\title{
Functional Near Infrared Spectroscopy in Novice and Expert Surgeons - A Manifold Embedding Approach
}

\author{
Daniel Richard Leff, Felipe Orihuela-Espina, Louis Atallah, Ara Darzi, \\ and Guang-Zhong Yang
}

\begin{abstract}
Royal Society/Wolfson Medical Image Computing Laboratory \& Department of Biosurgery and Surgical Technology, Imperial College London, United Kingdom \{d.leff, f.orihuela-espina, 1.atallah, a.darzi, g.z.yang\}@imperial.ac.uk
\end{abstract}

\begin{abstract}
Monitoring expertise development in surgery is likely to benefit from evaluations of cortical brain function. Brain behaviour is dynamic and nonlinear. The aim of this paper is to evaluate the application of a nonlinear dimensionality reduction technique to enhance visualisation of multidimensional functional Near Infrared Spectroscopy (fNIRS) data. Manifold embedding is applied to prefrontal haemodynamic signals obtained during a surgical knot tying task from a group of 62 healthy subjects with varying surgical expertise. The proposed method makes no assumption about the functionality of the data set and is shown to be capable of recovering the intrinsic low dimensional structure of in vivo brain data. After manifold embedding, Earth Mover's Distance (EMD) is used to quantify different patterns of cortical behaviour associated with surgical expertise and analyse the degree of inter-hemispheric channel pair symmetry.
\end{abstract}

\section{Introduction}

Research evaluating technical skills in surgery is in a current state of flux. Traditional assessments of technical skills based on isolated evaluations of dexterity are slowly being replaced by combined analyses of visuo-motor strategies [1], hand-eye coordination [1] and more recently cortical brain function [2]. The motivation for the latter is to elucidate longitudinal performance variations, which may assist the selection and assessment of future surgeons. Cortical regions should be probed to determine whether excitation patterns vary according to technical expertise. This will inform assessments of neuroplasticity obtained through serial experiments. The prefrontal cortex, owing to its role in supporting acquisition of novel motor skills, is likely to be preferentially recruited in surgical novices [3].

Functional Near Infrared spectroscopy (fNIRS) is a non-invasive neuroimaging technique that permits evaluations of cortical function in ambulant subjects, enabling complex motor tasks to be evaluated in realistic settings and in real-time. NIR light (700-1000nm) emitted to the scalp is detectable as attenuated light following absorption and scattering on the cortical surface[4]. Attenuated light levels are interpreted as relative changes in oxygenated haemoglobin $\left(\mathrm{HbO}_{2}\right)$ and deoxygenated haemoglobin $(\mathrm{HHb})$; the two dominant chromophores in cortical vasculature. The 
typical haemodynamic response to cortical excitation consists of task induced $\mathrm{HbO}_{2}$ increases coupled to $\mathrm{HHb}$ decreases [5].

Multi-channel fNIRS data, with configuration illustrated in Figure 1, for example, is complex and its intrinsic dimension $d$ is given by $d=c \cdot u \cdot h \cdot s$, where $c$ are channels, $u$ are users or subjects, $h$ are number of haemoglobin components and $s$ are number of observations. Dimensionality reduction techniques may assist visualisation of fNIRS data. Brain signal behaviour is nonlinear and dynamic [6]. Principal Component Analysis (PCA) and Multi-dimensional Scaling (MDS) are problematic in capturing nonlinearities of the in vivo data. The purpose of this study is to improve the visualisation of prefrontal haemodynamic signals obtained from novice and expert surgeons, through the use of manifold embedding. The method is based on isometric feature mapping or Isomap for recovering the intrinsic dimensionality and nonlinear structure of the dataset [7]. The most important feature of the method is that it preserves the geodesic distances between local data points in the input space, which may better reflect their intrinsic similarity than the Euclidean distance. As opposed to studies in which the intrinsic structure of the data is known a priori [8], this study demonstrates the ability of the proposed method to recover meaningful global coordinates of data with unknown dimensions. Projecting the data into a low dimensional space enables cortical behaviour to be quantitatively compared between groups of observers, differing extensively in surgical expertise.

\section{Methods}

\subsection{Subjects and Task Paradigm}

Sixty-two, healthy right handed male subjects were recruited for this study (19 consultant surgeons, 21 surgical trainees, and 22 medical students). Medical students were trained in a single session to perform hand tied surgical reef knots. The paradigm investigated consists of four throws of a surgical reef knot. Following a period of baseline motor rest (30 seconds) prior to the start of the first stimulus, five experimental blocks were conducted, each consisting of 'trial' (self paced surgical reef knot) and 'post trial' rest periods (20-30 seconds). A previous feasibility study [2] demonstrated an appropriate signal to noise ratio, experiment duration and subject comfort, following five task repetitions.

\subsection{Functional NeuroImaging and Dexterity Analysis}

Optical measurements were acquired using a 24-channel Optical Topography System (ETG - 4000, Hitachi Medical Co., Japan). A configuration of two $3 \times 3$ probe arrays was used, composing ten optical fibre sources emitting NIR light (from laser diodes) at 690 and $830 \mathrm{~nm}$ and eight optical fibre receivers coupled to avalanche photodiode detectors [9]. Optodes were held within a plastic helmet (inter-optode distance $30 \mathrm{~mm}$ ), affixed to the participant's head with a surgical bandage. Optodes were placed bilaterally over the prefrontal cortices, positioned according to the International 10-20 system of electrode placement [10], as illustrated in Fig. 1. Detected light intensity data was sampled at $10 \mathrm{~Hz}$. Technical performance was monitored using a surgical assessment device [11], which translates positional data into dexterity measures. 


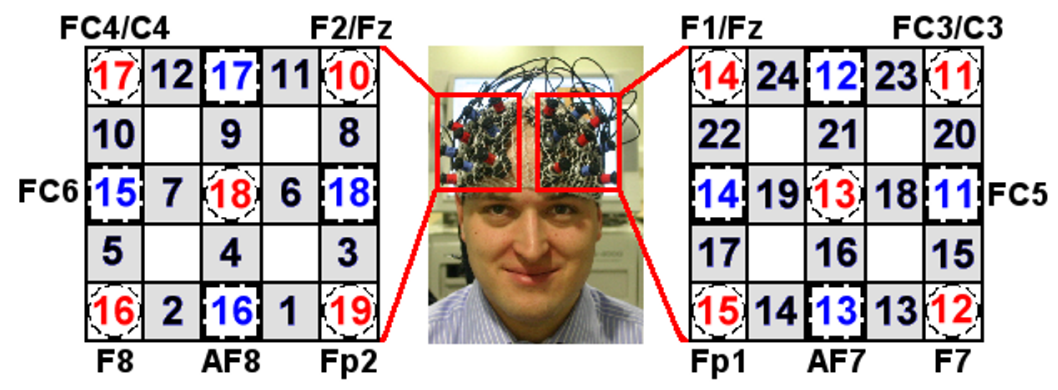

Fig. 1. Schematic illustration of the positions of NIR light sources (dotted circles), detectors (interrupted squares) and locations of corresponding channels numerically labelled (shaded boxes). The approximate 10/10 topographic location of each array is illustrated.

All optical data was processed using the functional Optical Signal Analysis program (fOSA, University College London, UK [12]). Relative changes in light intensities were converted to changes in haemoglobin $\left(\mathrm{HbO}_{2}, \mathrm{HHb}\right.$ and their sum, total haemoglobin HbT), applying the Modified Beer Lambert Law. Data was baseline corrected, decimated to $1 \mathrm{~Hz}$, and detrended to remove system drift and unrelated physiological signals. Data was averaged across experimental blocks. To overcome temporal variations in knot-tying durations, data was resampled applying a low pass filter. Final datasets comprised 20 baseline values, 37 trial values, and 20 post trial rest values for each NIR channel. Data was cleaned by visual assessment from two investigators blinded to each others results, followed by matching and agreement to eliminate noisy channels. A 74-D feature space $F$ was constructed by using resampled $\mathrm{HbO}_{2}$ and $\mathrm{HHb}$ trial values.

\subsection{Non Linear Dimensionality Reduction}

For manifold embedding, the Isomap algorithm [7] was used, which combined the algorithmic efficiencies of other dimensionality reduction techniques including global optimality, computational efficiency and guaranteed asymptotic convergence with the ability to learn a broad class of non-linear manifolds. Unlike other locally linear techniques, Isomap is able to represent the global structure of a dataset within a single coordinate system, which is particularly useful for group-wise comparison.

The three stages of the Isomap embedding begins with constructing the nearest neighbour graph in $F$ space based on Euclidian distances. The two standard approaches are to connect each point with its $\mathrm{K}$ nearest neighbours, or to connect all points within a radius $\varepsilon$. These neighbourhood relations are represented in a weighted graph $G$ over the data points, with edges of weight $d_{G}(i, j)$ between neighbouring points. In the second step, Isomap estimates the geodesic distances $d_{M}(i, j)$ between all pairs of points on the manifold $M$ by computing their shortest path distances in graph $G$. The matrix of the final values of $D_{G}$ contains the shortest path distances between all pairs of points in $G$. By applying classical MDS to matrix $D_{G}$ and constructing embedding data in $n$-dimensional Euclidean space $P$ that best preserves the intrinsic geometry, the embedded results is derived. MDS uses a function 
minimization algorithm which evaluates different possible arrangements of points in the $P$ space aiming to maximizing the goodness of fit. The cost function $E$ used by Isomap is given by the $\mathrm{L} 2$ norm of the difference expressed as:

$$
E=\left\|\tau\left(D_{G}\right)-\tau\left(D_{P}\right)\right\|
$$

where $D_{P}$ is the matrix of Euclidean distances in $P$ space, and $\tau$ is an operator to convert the distances matrix to scalar products.

\subsection{Earth Mover's Distance (EMD)}

For assessing cortical excitation patterns of different subject groups, EMD is used as a metric for quantitative comparison. EMD is based on the transportation problem [13] and evaluates the dissimilarity between two multidimensional distributions, which has been used to analyse visual search behaviours using eye-tracking[14]. Each distribution is composed of a set of points each one with an associated weight $w$. This is referred to as the signature of the distribution on the manifold. The EMD between two signatures is the minimum amount of 'work' needed to transform one signature into another. The work required is the proportion of weight being moved, multiplied by the distance between the old and new locations. Consider two distributions $X=\left\{\left(x_{1}, w_{x 1}\right),\left(x_{2}, w_{x 2}\right), \ldots,\left(x_{m}, w_{x m}\right)\right\}$ and $Y=\left\{\left(y_{1}, w_{y 1}\right),\left(y_{2}, w_{y 2}\right), \ldots,\left(y_{n}, w_{y n}\right)\right\}$. It is possible to define a cost matrix $C$ such that $c_{i j}$ represents the cost of transforming a point $i=1 \ldots m$ into a point $j=1 . . n$ (known as ground distance). Mathematically, EMD can be defined as:

$$
\operatorname{EMD}(X, Y)=\frac{\sum_{i=1}^{m} \sum_{j=1}^{n} c_{i j} f_{i j}}{\sum_{i=1}^{m} \sum_{j=1}^{n} f_{i j}}
$$

where each $f_{i j}$ is a flow representing the amount of weight moved from $i$ to $j$. The ground distances are the $\mathrm{L}_{2}$ norm, and hence EMD is a lower bounded metric.

EMD aims to find a flow set $\mathbf{f}=\left\{f_{i j}\right\}$ subject to the following constraints:

$$
\begin{gathered}
f_{i j} \geq 0, \quad 1 \leq i \leq m, 1 \leq j \leq n \\
\sum_{i=1}^{m} f_{i j} \leq w_{y j}, \quad j=1 \ldots n \\
\sum_{j=1}^{n} f_{i j} \leq w_{x i}, \quad i=1 \ldots m \\
\sum_{i=1}^{m} \sum_{j=1}^{n} f_{i j}=\min \left(\sum_{i=1}^{m} w_{x i}, \sum_{j=1}^{n} w_{y j}\right)
\end{gathered}
$$

That minimises function $\sum_{i=1 \ldots m} \sum_{j=1 \ldots n} c_{i j} f_{i j}$ 


\section{Results}

Typical haemodynamic responses to knot-tying are illustrated in Fig. 2. Each subject produces as many points in the 74 dimensional feature space $F$ as there are channels, i.e. 24 in this study. Isomap was used to map points from $F$ to $P$ using $k=7$ neighbours to construct graph $G$. The intrinsic dimensionality, as estimated from the flexure point of the residual variance was located at the third component. The ability of the embedding technique to resolve meaningful global co-ordinates is highlighted in Fig. 3, where each component of the embedding correlates well with one degree of freedom of the underlying data, left to right, $\mathrm{HbO}_{2}\left(r^{2}=0.97\right)$, up and down, $\mathrm{HHb}$ $\left(r^{2}=0.65\right)$. Fig. 4 further illustrates the results of two-dimensional embedding for each individual NIR channel. It is evident that consultant surgeons and trainees appear tightly clustered in $P$, whereas greater dispersion is observed in novices.
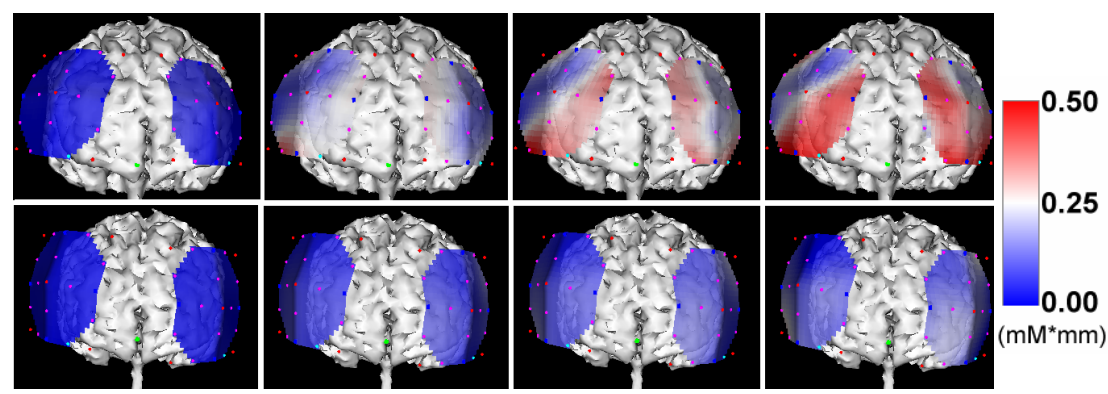

Fig. 2. Topographic maps illustrating $\mathrm{HbO}_{2}$ intensity change observed in a novice (top row) and consultant (bottom row). Columns represent baseline rest, 2, 6, and 10 seconds into a knottying trial. Emitters (red circles), detectors (blue circles) and corresponding channels (pink circles) are overlaid on to T1 weighted anatomical MRI images.

Once data is mapped to the embedded space, EMD was used to compare distributions between groups for a specific channel of interest, as well as to compare a given channel and its symmetrical opposite in the contralateral hemisphere. In both instances, the Euclidean distances between points in $P$ space were used as the ground distance. Euclidean distances in the embedded space $P$ are an approximation of the geodesic distance in $F$ space. EMD weights are calculated as $1 / z$ with $z$ being the number of points in the single cluster of each signature.

Table 1 summarises the relevant EMD results for comparison between groups. Greater similarity is observed between trainees and consultants, than between these groups and novices. The degree of inter-hemispheric channel pair symmetry is illustrated in Fig. 5 (a). In order to contextualize inter-hemispheric symmetry in terms of cortical excitation, further analysis was performed specifically comparing averaged resting $\mathrm{Hb}$ values with averaged stimulus values using non-parametric tests of significance (Mann Whitney U). The results are illustrated in Fig. 5 (b). In novices, patterns consistent with 'activation' were only observed in left hemispheric channels, indicating an asymmetrical response. 

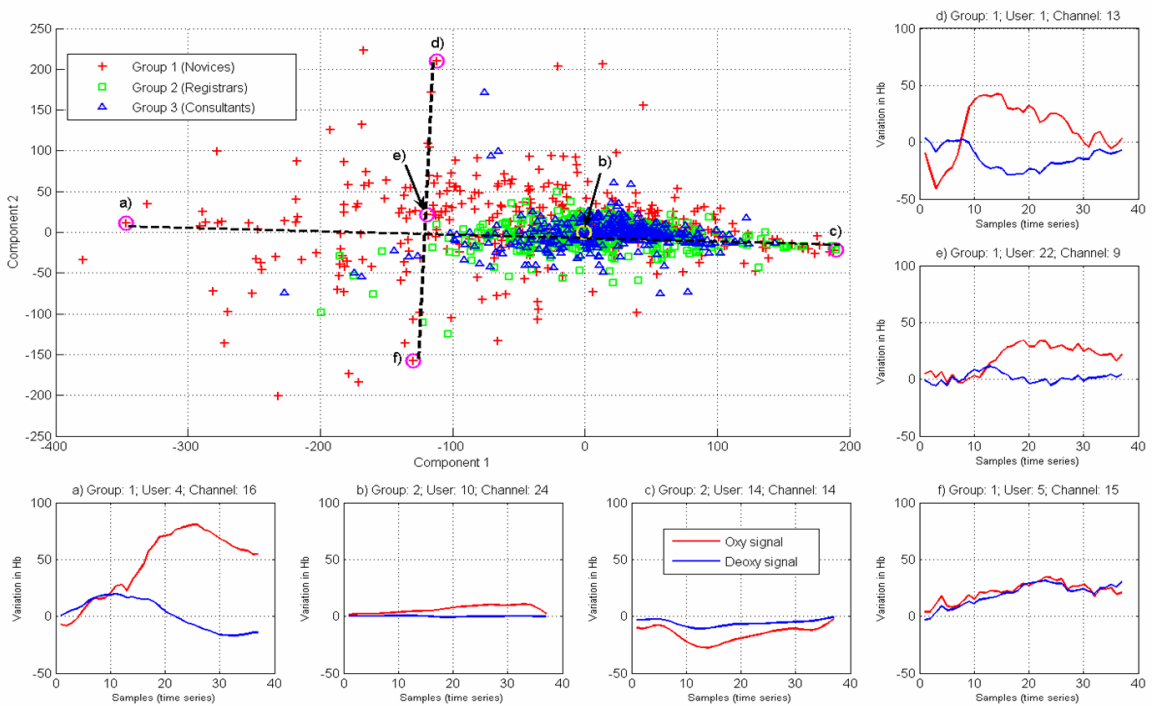

Fig. 3. Manifold embedding results showing the distribution of channel response and how it varies along the first two principal dimensions for all the subjects studied. The locations of the original signals (a-f), representing resampled task related $\mathrm{Hb}$ signals, are marked on the embedded space. This demonstrates the intrinsic trend captured by the embedding technique.

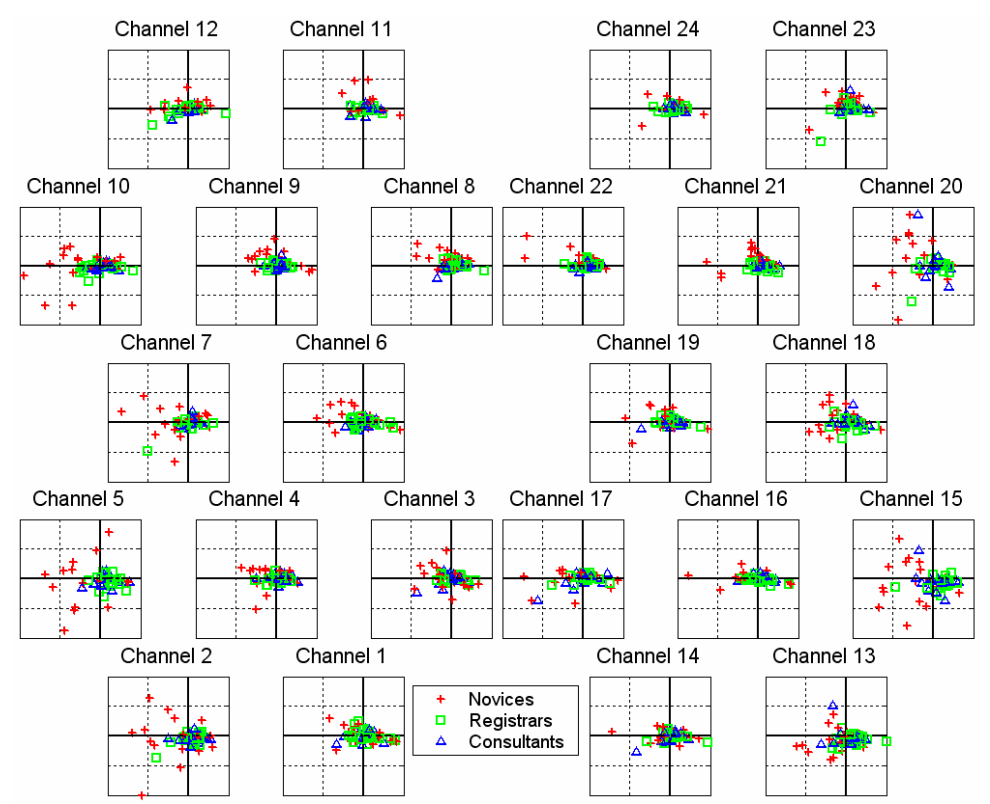

Fig. 4. Embedding per NIR channel where novices (Group 1) are labelled as plus signs, trainees (Group 2) as squares and consultants (Group 3) as open triangles. All subplots are constructed on identical scales. The abscissa corresponds to Component 1 and the ordinate to Component 2. 
Table 1. Comparison of between-group EMD results

\begin{tabular}{cccc}
\hline Parameter & $\begin{array}{c}\text { Novices to } \\
\text { Consultants }\end{array}$ & $\begin{array}{c}\text { Novices to } \\
\text { Trainees }\end{array}$ & $\begin{array}{c}\text { Trainees to } \\
\text { Consultants }\end{array}$ \\
\hline Mean & 81.62 & 79.50 & 24.19 \\
\hline St.Dev & 32.62 & 39.53 & 6.88 \\
\hline
\end{tabular}

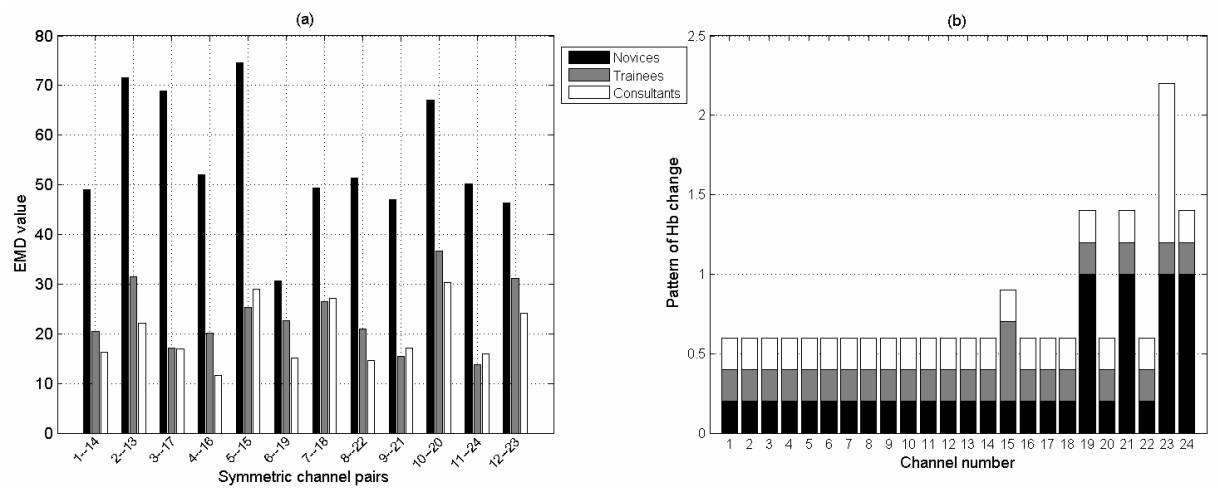

Fig. 5. (a) Illustration of inter-hemispheric channel pair symmetry as quantified using EMD. (b) Task induced $\mathrm{Hb}$ changes across all 24 channels. Significant $(p \leq 0.05)$ increases in $\mathrm{HbO}_{2}$ and decreases in $\mathrm{HHb}$ were assigned 1.0. All other patterns of change were assigned 0.2 except for nonsignificant $\mathrm{HbO}_{2}$ changes, which were assigned 0.5.

Knot tying performance data, as measured by ICSAD, was analysed to determine if dissimilarity in cortical signal behaviour was associated with variations in surgical expertise. Dexterity data was analysed using non-parametric tests of significance. For each dexterity measure of interest, comparisons between groups were made using the Mann Whitney $\mathrm{U}$ test. A value of $p \leq 0.05$ was assumed to be statistically significant. Consultants and trainees were significantly faster $(p \leq 0.001)$, made fewer unnecessary movements $(p \leq 0.001)$ and used shorter path-lengths $(p \leq 0.001)$ to complete trials compared to medical students.

\section{Discussion and Conclusion}

In this paper, a novel approach to understanding complex fNIRS data has been proposed. The technique places no assumption regarding the functionality of the dataset, yet is capable of identifying meaningful patterns of cortical signal behaviour in subjects with different surgical experience. The results suggest that patterns of prefrontal behaviour are comparable in consultants and trainees, in whom technical performance could not be discriminated. In contrast, medical students exhibit dissimilar patterns of cortical behaviour and significantly poorer performance. Moreover, EMD results strongly suggest an asymmetrical cortical response, most pronounced in novices. This is validated by conventional statistical analysis, which 
demonstrated lateralised left prefrontal excitation in medical students. Future work should aim to classify patterns of cortical activation in low-dimensional space, and to clarify the functional significance of hemispheric lateralisation. It is anticipated that these techniques may discriminate observers based upon surgical expertise.

\section{References}

1. Leong, J.J.H., Nicolaou, M., Atallah, L., Mylonas, G., Darzi, A.W., Yang, G.Z.: HMM Assessment of Quality of Movement Trajectory in Laparoscopic Surgery. In: Larsen, R., Nielsen, M., Sporring, J. (eds.) MICCAI 2006. LNCS, vol. 4190, pp. 752-759. Springer, Heidelberg (2006)

2. Leff, D.R., Koh, P.H., Aggarwal, R., Deligiani, F., Elwell, C., Delpy, D.T., Darzi, A.W., Yang, G.Z.: Optical Mapping of the Frontal Cortex During a Surgical Knot-Tying Task, a Feasibility Study. In: Medical Imaging Augmented Reality (MIAR), vol. 4091, pp. 140 147 (2006)

3. Halsband, U., Lange, R.K.: Motor learning in man: a review of functional and clinical studies. J. Physiol. Paris 99, 414-424 (2006)

4. Villringer, A., Planck, J., Hock, C., Schleinkofer, L., Dirnagl, U.: Near infrared spectroscopy (NIRS): a new tool to study hemodynamic changes during activation of brain function in human adults. Neurosci. Lett. 154, 101-104 (1993)

5. Strangman, G., Boas, D.A., Sutton, J.P.: Non-invasive neuroimaging using near-infrared light. Biol. Psychiatry 52, 679-693 (2002)

6. Friston, K.J.: Human Brain Fuction, 2nd edn. (2003)

7. Tenenbaum, J.B., de Silva, V., Langford, J.C.: A global geometric framework for nonlinear dimensionality reduction. Science 290, 2319-2323 (2000)

8. Geng, X., Zhan, D.C., Zhou, Z.H.: Supervised Nonlinear Dimensionality Reduction for Visualization and Classification. IEEE Transactions on systems, man and cybernetics, Part B: cybernetics 35, 1098-1107 (2005)

9. Sato, H., Kiguchi, M., Kawaguchi, F., Maki, A.: Practicality of wavelength selection to improve signal-to-noise ratio in near-infrared spectroscopy. NeuroImage 21, 1554-1562 (2004)

10. Jasper, H.H.: The ten-twenty electrode system of the International Federation. Electroencephalogr. Clin.Neurophysiol. 10, 371-375 (1958)

11. Taffinder, N., Smith, S., Mair, J., Russell, R., Darzi, A.: Can a computer measure surgical precision? Reliability, validity and feasibility of the ICSAD. Surg. Endosc. 13, 81 (1999)

12. Koh, P.H., Delpy, D.T., Elwell, C.E.: fOSA: A software tool for NIRS processing. In: Proceedings of Optical Society of America (2006)

13. Rubner, Y., Tomasi, C., Guibas, L.J.: A metric for distributions with applications to image databases. In: ICCV 1998. International Conference on Computer Vision, pp. 59-66 (1998)

14. Dempere-Marco, L., Hu, X.P., Ellis, S.M., Hansell, D.M., Yang, G.Z.: Analysis of visual search patterns with EMD metric in normalized anatomical space. IEEE Trans. Med. Imaging. 25, 1011-1021 (2006) 